were made on sections at various distances from the fixed end of the beam. As extreme values they found $y_{0}=0.857 d$ and $y_{0}=0.814 d$. Formula (7) with $\beta=0.041$ and $\sigma=0.27$ gives $y_{0}=0.8414 d$, a value which agrees well with the experimental results.

Iowa State College of Agriculture and Mechanic Arts

\title{
ON THE METRIZATION OF UNIFORM SPACE
}

\section{W. COHEN AND CASPER GOFFMAN}

We call a topological space $S$ metrizable by a group $G$ if there is a distance function for $S$ with values in $G$ which defines an equivalent metric topology in $S$. It should be noted that for the triangle inequality required of a distance to have meaning, the group $G$ must be ordered, at least partially. In the classical metrization theorem of Chittenden $[1],{ }^{1} G$ is the additive group of real numbers. The form of this theorem due to Weil [2] is that a necessary and sufficient condition for a uniform space to be metrizable by the group of real numbers is that it satisfy uniformly the first denumerability axiom of Hausdorff. Kalisch [3], using Weil's theorem that a uniform space is equivalent to a subset of a direct product of metric spaces with real distances, showed that every uniform space is metrizable by a partially ordered group. A related result due to Zelinsky [4] gives necessary and sufficient conditions that a topological field have a valuation in an ordered group.

Here we give necessary and sufficient conditions that a uniform space be metrizable by an ordered abelian group $G$. We shall assume that the identity in $G$ is not isolated in the order of $G$, a restriction which excludes the topologically uninteresting discrete spaces. We have shown [5] that the topology of such a group $G$ is determined by a limiting ordinal $\xi^{*}=\xi^{*}(G)$ with the property that if $\eta^{*}<\xi^{*}$ and $\xi_{\eta}$ is a single-valued function on $\eta<\eta^{*}$ to $\xi<\xi^{*}$, then

$$
\sup \left[\xi_{\eta} \mid \eta<\eta^{*}\right]<\xi^{*} ;
$$

and elements $a_{\xi}, \xi<\xi^{*}$, decreasing in $G$, such that inf $\left[a_{\xi} \mid \xi<\xi^{*}\right]=\theta$, the identity in $G$.

THEOREM. A uniform space $S$ is metrizable by an ordered abelian

Presented to the Society, October 29, 1949; received by the editors September 9, 1949.

${ }^{1}$ Numbers in brackets refer to the bibliography at the end of the paper. 
group $G$ if and only if $S$ has a neighborhood system $V=\left[U_{\xi}(x), \xi<\xi^{*}\right.$, $x \in S]$ with the properties

1. $\bigcap_{\xi<\xi} U_{\xi}(x)=[x]$,

2. $\xi_{1}<\xi_{2}<\xi^{*}$ implies $U_{\xi_{1}}(x) \supset U_{\xi_{2}}(x)$,

3. $\eta<\xi^{*}$ implies there is a $\xi(\eta)$ such that $\eta \leqq \xi(\eta)<\xi^{*}$ and if $U_{\xi(\eta)}(x)$ $\cap U_{\xi(\eta)}(y) \neq 0$, then $U_{\xi(\eta)}(y) \subset U_{\eta}(x)$,

$4^{\prime} . \eta^{*}<\xi^{*}$ implies that $\bigcap_{\eta<\eta^{*}} U_{\xi(\eta)}(x)$ is open, where $\xi^{*}$ is a limiting ordinal with the property (*).

PROof. As a preliminary, we remark that, since $\omega$ is the first limiting ordinal with the property $\left({ }^{*}\right)$, we may restrict ourselves to the case $\xi^{*}>\omega$ in view of the classical metrization theorem.

For the necessity: Suppose that $d(x, y)$ is a distance function for $S$ with values in $G$. The metric topology in $S$ is defined by the spheres $S(x, \xi)=\left[y \mid d(x, y)<a_{\xi}\right], \xi<\xi^{*}$. Property 1 holds since inf $a_{\xi}=\theta$; property 2 holds since the $a_{\xi}$ decrease on $\xi<\xi^{*}$; property 3 holds since $d(x, y)$ has the triangle property of a distance function; property $4^{\prime}$ holds since $\xi^{*}$ is a limiting ordinal with property $\left({ }^{*}\right)$.

For the sufficiency: First we show that $S$ has a neighborhood system $\mathscr{W}=\left[V_{\xi}(x)\right]$, equivalent to $U$, with properties $1,2,3,4^{\prime}$, and

5. $V_{\xi}(x) \cap v_{\xi}(y) \neq 0$ implies $V_{\xi}(x)=V_{\xi}(y)$.

For $\eta<\xi^{*}$ we put $\eta_{0}=\eta$ and $\eta_{n}=\xi\left(\eta_{n-1}\right), n<\omega$, where $\xi\left(\eta_{n-1}\right)$ is the ordinal of 3 . Since $\omega<\xi^{*}, \lambda(\eta)=$ sup $\left[\eta_{n} \mid n<\omega\right]<\xi^{*}$. We define $\zeta(\eta)$ $=\sup [\lambda(\mu) \mid \mu<\eta+1]$. From 3 and $\left(^{*}\right)$ we have $\eta \leqq \xi(\eta) \leqq \lambda(\eta) \leqq \zeta(\eta)$ $<\xi^{*}$ and $\zeta\left(\eta_{1}\right) \leqq \zeta\left(\eta_{2}\right)$ if $\eta_{1}<\eta_{2}$. We define $V_{\eta}(x)$ as the set of $y$ for which there are $n<\omega$ and $x_{1}=x, x_{2}, \cdots, x_{n}=y \in S$ such that $U_{\zeta(n)}\left(x_{k}\right)$ $\cap U_{\zeta(n)}\left(x_{k+1}\right) \neq 0$ for $k=1, \cdots, n-1$.

The equivalence of $\mathscr{W}=\left[V_{\eta}(x)\right]$ and $U$ is established by showing that $U_{\xi(\eta)}(x) \subset V_{\eta}(x) \subset U_{\eta}(x), \eta<\xi^{*}$. The first inclusion follows from $y \in U_{\zeta(\eta)}(x)$ and $x_{1}=x, x_{2}=y$. For the second, consider $y \in V_{\eta}(x)$. Then there are $n<\omega$ and $x_{1}=x, x_{2}, \cdots, x_{n}=y \in S$ such that $U_{\zeta(\eta)}\left(x_{k}\right)$ $\cap U_{\zeta(\eta)}\left(x_{k+1}\right) \neq 0, k=1, \cdots, n-1$. From 2 and $\eta_{k} \leqq \lambda(\eta) \leqq \zeta(\eta)$, we have $U_{\eta_{k}}\left(x_{k}\right) \cap U_{\eta_{k}}\left(x_{k+1}\right) \neq 0$. Since $\eta_{k}=\xi\left(\eta_{k-1}\right)$ we have, from 3 , $U_{\eta_{k}}\left(x_{k+1}\right) \subset U_{\eta_{k-1}}\left(x_{k}\right)$. Hence

$$
y=x_{n} \in U_{\eta_{n-1}}\left(x_{n}\right) \subset U_{\eta_{n-2}}\left(x_{n-1}\right) \subset \cdots \subset U_{\eta_{0}}\left(x_{1}\right)=U_{\eta}(x)
$$

and so $V_{\eta}(x) \subset U_{\eta}(x)$.

That the system $W$ has properties 1 and $4^{\prime}$ follows from its equivalence with $U$. For property 2 consider $\eta^{\prime}<\eta^{\prime \prime}$. If $y \in V_{\eta^{\prime \prime}}(x)$, then for some $x_{1}=x, x_{2}, \cdots, x_{n}=y, U_{\zeta\left(\eta^{\prime \prime}\right)}\left(x_{k}\right) \cap U_{\zeta\left(n^{\prime \prime}\right)}\left(x_{k+1}\right) \neq 0, k=1, \cdots$, $n-1$. Since $U$ has property 2 and $\zeta\left(\eta^{\prime}\right) \leqq \zeta\left(\eta^{\prime \prime}\right), U_{\zeta\left(\eta^{\prime}\right)}\left(x_{k}\right) \cap U_{\zeta\left(\eta^{\prime}\right)}\left(x_{k+1}\right)$ $\neq 0$. Hence $y \in V_{\mathbf{y}^{\prime}}(x)$ and $\mathscr{W}$ has property 2 . For property 3 consider 
$\eta<\xi^{*}$ and the ordinal $\xi(\zeta(\eta))$ corresponding to $\zeta(\eta)$ by property 3 for the system $U$. Now by the inclusions of the equivalence argument, $V_{\xi(\zeta(\eta))}(y) \cap V_{\xi(\zeta(\eta))}(x) \neq 0$ implies $U_{\xi(\zeta(\eta))}(y) \cap U_{\xi(\zeta(\eta))}(x) \neq 0$ and so $V_{\xi(\zeta(\eta))}(y) \subset U_{\xi(\zeta(\eta))}(y) \subset U_{\zeta(\eta)}(x) \subset V_{\eta}(x)$. Since $\eta \leqq \zeta(\eta) \leqq \xi(\zeta(\eta))$, $\xi(\zeta(\eta))$ serves as the ordinal corresponding to $\eta$ in property 3 for $\mathscr{W}$.

For property 5 consider $w \in V_{\eta}(x) \cap V_{\eta}(y)$ and $z \in V_{\eta}(y)$. Then there are $x_{1}^{\prime}=x, x_{2}^{\prime}, \cdots, x_{p}^{\prime}=w ; y_{1}^{\prime}=y, y_{2}^{\prime}, \cdots, y_{q}^{\prime}=w ; y_{1}^{\prime \prime}=y, y_{2}^{\prime \prime}, \cdots$, $y_{r}^{\prime \prime}=z$ in $S$ such that $U_{\zeta(\eta)}\left(u_{k}\right) \cap U_{\zeta(\eta)}\left(u_{k+1}\right) \neq 0$ if $u_{k}=x_{k}^{\prime}, k=1, \cdots$, $p-1 ; u_{k}=y_{k}^{\prime}, k=1, \cdots, q-1 ; u_{k}=y_{k}^{\prime \prime}, k=1, \cdots, r-1$, respectively. We now have $x_{1}=x, x_{2}, \cdots, x_{p+q+r-2}=z$ and $U_{\zeta(\eta)}\left(x_{k}\right)$ $\cap U_{\zeta(\eta)}\left(x_{k+1}\right) \neq 0, k=1, \cdots, p+q+r-3$. Hence $z \in V_{\eta}(x)$ and 5 follows easily.

The ordered group $G$ by which $S$ is metrizable is the additive group of real functions on the ordinals $\xi<\xi^{*}$. For: Given $x, y \in S$ and $\xi<\xi^{*}$ we define

$$
d_{\xi}(x, y)= \begin{cases}0, & V_{\xi}(x)=V_{\xi}(y), \\ 1, & V_{\xi}(x) \neq V_{\xi}(y) .\end{cases}
$$

For each pair $x, y \in S$ the $d_{\xi}(x, y)$ are the values of a real function $d(x, y)$ on $\xi<\xi^{*}$. It is clear that $d(x, y)=d(y, x) \geqq \theta$, the identity in $G$. Since $\mathscr{W}$ has property $1, d(x, y)=\theta$ if and only if $x=y$. For the triangle property of $d(x, y)$, consider $x, y, z \in S$ and $\xi<\xi^{*}$ such that $d_{\xi}(x, z)$ $=d_{\xi}(z, y)=0$. Then $V_{\xi}(x)=V_{\xi}(z)=V_{\xi}(y)$ and so $d_{\xi}(x, y) \leqq d_{\xi}(x, z)$ $+d_{\xi}(z, y)$.

Now by the definition of order in $G$ we have $d(x, y) \leqq d(x, z)$ $+d(z, y)$ for all $x, y, z \in S$. Hence $d(x, y)$ is a distance function for $S$ with values in $G$.

Finally we show that $S$ is metrizable by the group $G$. Let $a_{\xi} \in G$, $\xi<\xi^{*}$, be the functions

$$
a_{\xi}(\eta)= \begin{cases}1, & \eta=\xi \\ 0, & \eta \neq \xi\end{cases}
$$

For each $\xi<\xi^{*}$ and $x \in S, V_{\xi}(x)=\left[y \mid d(x, y)<a_{\xi}\right]=S(x, \xi)$. For: Consider $y \in V_{\xi}(x)$. By property $2, y \in V_{\eta}(x), \eta \leqq \xi$, and so, by property $5, d_{\eta}(x, y)=0$ for $\eta \leqq \xi$. Hence $d(x, y)<a_{\xi}$. Conversely, if $d(x, y)$ $<a_{\xi}, d_{\eta}(x, y)=0$ for $\eta \leqq \xi$ and so $V_{\xi}(x)=V_{\xi}(y)$. From property 1 it follows that $y \in V_{\xi}(x)$.

We conclude with 3 remarks: 1 . If $\xi^{*}>\omega$, the metric for $S$ is nonarchimedean. 2. The spaces considered in [6] are metrizable by ordered abelian groups. 3. The space of real functions of a real variable with the weak topology is not metrizable by an ordered abelian group. 


\section{BIBLIOGRAPHY}

1. E. W. Chittenden, The metrization problem, Bull. Amer. Math. Soc. vol. 33 (1927) pp. 13-34.

2. A. Weil, Sur les espaces d structures uniforme, Paris, Herman, 1938.

3. G. K. Kalisch, On uniform spaces and topological algebra, Bull. Amer. Math. Soc. vol. 52 (1946) pp. 936-939.

4. D. Zelinsky, Topological characterization of fields with valuations, Bull. Amer. Math. Soc. vol. 54 (1948) pp. 1145-1150.

5. L. W. Cohen and Casper Goffman, The topology of ordered abelian groups, Trans. Amer. Math. Soc. vol. 67 (1949) pp. 310-319.

6. - A theory of transfinite convergence, Trans. Amer. Math. Soc. vol. 66 (1949) pp. 65-74.

Queens College and

UNIVERSITY OF OKLAHOMA 